\title{
De novo isochromosome $18 p$ in a female dysmorphic child
}

\author{
Smitha Ramegowda ${ }^{1}$, Harshavardhan M. Gawde ${ }^{2}$, Abbas Hyderi $^{3}$, Mysore R. Savitha ${ }^{3}$, \\ Zareen M. Patel ${ }^{2}$, Balasundaram Krishnamurthy ${ }^{3}$, Nallur B. Ramachandra ${ }^{1}$ \\ ${ }^{1}$ Human Genetics Laboratory, Department of Studies in Zoology, University of Mysore, Manasagangothri, Mysore, India \\ ${ }^{2}$ National Institute for Research in Reproductive Health, Genetic Research Center (GRC-ICMR), J.M. Street, Parel, Mumbai, India \\ ${ }^{3}$ Department of Pediatrics, Cheluvamba hospital, Mysore, India
}

\begin{abstract}
Isochromosome 18p results in tetrasomy 18p. Most of the i $(18 \mathrm{p})$ cases reported so far in the literature are sporadic due to de novo formation, while familial and mosaic cases are infrequent. It is a rare chromosomal abnormality, occurring once in every 140000 livebirths, affecting males and females equally. In the present investigation, we report a de novo i(18p) in a female dysmorphic child. The small metacentric marker chromosome was confirmed as $\mathrm{i}(18 \mathrm{p})$ in the proband by cytogenetic and FISH analysis [47,XX $+\mathrm{i}(18 \mathrm{p})]$. Cytogenetic investigations in the family members revealed normal chromosome numbers, indicating the case as a de novo event of $\mathrm{i}(18 \mathrm{p}$ ) formation. It could be due to the somewhat advanced maternal age (32 years) and/or expression of recessive genes in the proband, who is the progeny of consanguineous marriage, which could have led to misdivision and nondisjunction of chromosome 18 in meiosis I, followed by failure in the chromatid separation of $18 \mathrm{p}$ in meiosis II and by inverted duplication.
\end{abstract}

Key words: consanguineous parents, de novo, isochromosome, i(18p), nondisjunction, recessive genes.

Isochromosomes are the supernumerary marker chromosomes, which are made up of 2 copies of the same arm of a chromosome containing 1-2 centromeres, so that they form a mirror image of each other, resulting in a tetrasomy of the arm involved (Irwin et al. 2003). Prevalence of various kinds of isochromosomes ranges from 0.14 to 0.72 per 1000 live births (Rothlisberger et al. 2000).

Isochromosome $18 \mathrm{p}$ results in tetrasomy $18 \mathrm{p}$ (Rivera et al. 1984; Callen et al. 1990). The first case of $\mathrm{i}(18 \mathrm{p})$ was reported by Froland et al. (1963), which was followed by reports on few familial cases (Taylor et al. 1975; Takeda et al. 1989) and few mosaic cases of $i(18 p)$ (Gocke et al. 1986; Abeliovich et al. 1993; Back et al. 1994; Pinto et al. 1998). The majority of the i(18p) cases reported so far are sporadic due to de novo formation (Fujita and Fujita 1975; Fryns et al. 1990; Singer et al. 1990; Back et al. 1994; Eggermann et al. 1996). However, most of the reported familial cases of $i(18 p)$ were found to be of maternal or- igin (Kotzot et al. 1996; Bugge et al. 1996; Irwin et al. 2003), with only one case of paternal origin (Eggermann et al. 1997). It is a rare chromosomal abnormality with few nonmosaic cases reported as surviving beyond 2 years of age (Irwin et al. 2003). Isochromosome $18 p$ is found once in every 140000 live births, affecting males and females equally (http://www.chromosome18.org/syndromes.html).

The tetrasomy $18 p$ syndrome most often expresses itself with moderate to severe mental impairment, very delayed speech, poor or nonexistent ability to self feed, much delayed ability to walk, microcephaly, congenital heart diseases, etc. (Singer et al. 1990; Yu et al. 1993; Back et al. 1994; Eggermann et al. 1997). The conventional banding technique allows only a first step of diagnosis of isochromosomes, whereas fluorescence in situ hybridization (FISH) is the most common, rapid and reliable technique used to delineate isochromosomes. Chromosome 18p specific FISH probes for the identification of $i(18 p)$ was pro- 
posed for the first time in 1990 by Callen et al. Later on, several cases of $\mathrm{i}(18 \mathrm{p})$ have been reported by using centromeric, whole chromosome painting and arm-specific FISH probes (Abeliovich et al. 1993; Eggermann et al. 1997; Pinto et al. 1998; Rothlisberger et al. 2000). In the present investigation, we report a de novo $\mathrm{i}(18 \mathrm{p})$ in a female dysmorphic child. tal heart disease. Echocardiography revealed that there was no major congenital heart defect, but Doppler imaging revealed slight tricuspid regurgitation, trivial pulmonary regurgitation and systolic pulmonary artery pressure by TR JET: $38 \mathrm{mmHg}$. Pedigree analysis of the proband's family revealed that the proband was the third liveborn child of consanguineous parents (first-cousin marriage).
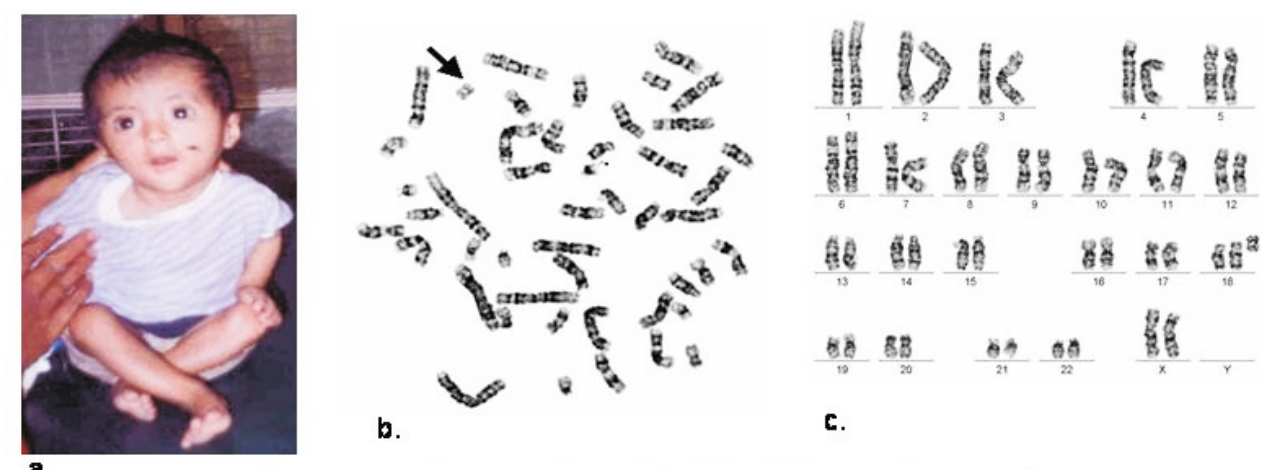

a.

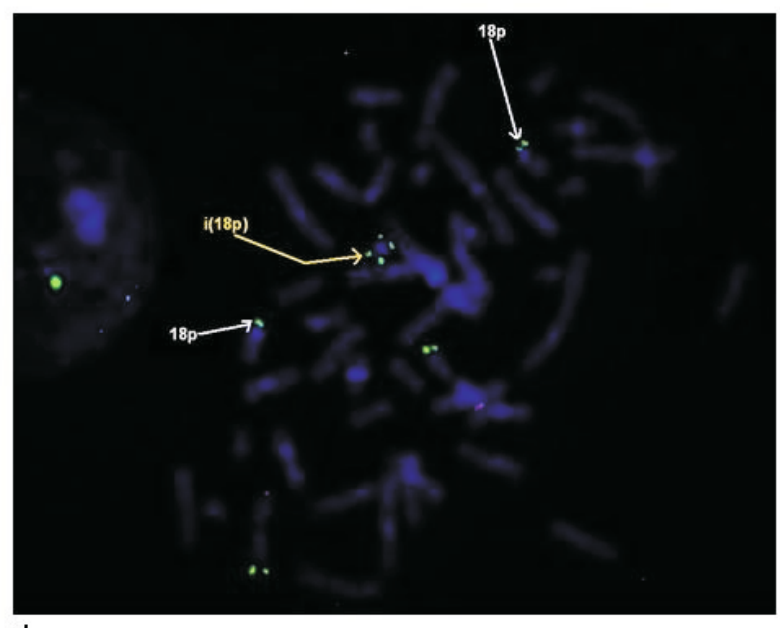

d.

Figure 1. (a) Proband at the age of 9 months, showing clinically microcephaly, high nasal bridge, small mouth, small nose, long distance from top of lip to bottom of nose, slender body, long face, eyes far apart, and poor muscle strength; (b) G-banded metaphase plate of the proband showing isochromosome 18p (arrow); (c) Karyotype of the metaphase plate (b) with isochromosome 18p; (d) FISH plate showing the subtelomeric region of chromosome 18p on metaphase chromosome of the tetrasomy $18 \mathrm{p}$ child. FISH probe identifies chromosome 18p (spectrum green), centromeric 18 (spectrum aqua), chromosome 11p (spectrum green) and chromosome 11q (spectrum orange). The labels and arrows denote the $18 \mathrm{p}$ of the normal chromosome 18 (white) and isochromosome $18 \mathrm{p}$ (yellow), respectively.

The proband (Figure 1a) was delivered by caesarean section at 38 weeks 3 days after an uneventful pregnancy, with a birth weight of $2000 \mathrm{~g}$. At 10 months of age the proband was brought to Cheluvamba hospital, Mysore, with complaints like hurried breathing, nasal discharge, on-and-off fever, and constipation (passing hard stools once a week). Clinical investigations revealed body weight below the 5 th percentile for age and sex, short stature, global delay in gross motor, fine motor, personal social and language milestones, microcephaly, depressed nasal bridge, epicanthal folds, low-set ears, high-arched palate, clinodactyly, and congeni-
At the time of conception of the proband, the age of the mother and father was 32 and 36 years, respectively. There was no history of birth defects or genetic defects in the family. However, the mother had suffered miscarriage twice, during the third month of the second conception and fifth month of the fourth conception. For further investigations the proband was referred for cytogenetic analysis.

Chromosomal analysis of the proband and family members with their consent (father, mother, elder sister and brother) was carried out on peripheral blood lymphocyte culture by using 
the standard protocol of Seabright (1971), with slight modifications. G-banded metaphases were screened by using a Leica DMRA2 research microscope. A total of 50 well-banded metaphase plates were analyzed and karyotyped according to the International System for Human Cytogenetic Nomenclature (ISCN 1995).

Telomeric FISH (T-FISH) was performed by using a ToTELVYSION subtelomeric probe (VYSIS, France, cat. No. 33-270000). The mixture contained probes for the subtelomeric region of chromosome $18 \mathrm{p}$ (spectrum green), centromeric 18 (spectrum aqua), chromosome $11 \mathrm{p}$ (spectrum green) and chromosome 11q (spectrum orange). T-FISH was performed according to the manufacturer's instructions. A total of 50 metaphase plates were analyzed.

The cytogenetic analysis ( $\mathrm{G}$ banding) of the proband revealed $47, \mathrm{XX},+\mathrm{i}(18 \mathrm{p})$ in all the 50 metaphase plates screened (Figure $1 \mathrm{~b}$ and $\mathrm{c}$ ), while all other members of the family had normal karyotypes, indicating the case as a de novo event of i $(18 p)$ formation. Further, FISH analysis has confirmed the marker chromosome to contain chromosome 18p material (Figure 1d) in all the metaphase plates. The hybridization of chromosome-18-specific centromeric probe shows that the $\mathrm{i}(18 \mathrm{p})$ is monocentric. Analyses of a total of 50 cells confirmed that all the metaphase plates are with the $i(18 p)$, suggesting it to be a nonmosaic case.

The proband in the present investigation has shown only a few characteristic clinical features consistent with the earlier reported cases of tetrasomy 18p (Singer et al. 1990; Yu et al. 1993; Back et al. 1994; Eggermann et al. 1997). This great variability of phenotypes could be a result of the different genetic content of the $i(18 p)$ in the present case. The cytogenetic investigation in the proband revealed a small metacentric chromosome by conventional $\mathrm{G}$ banding method, which was further confirmed by the FISH technique as $\mathrm{i}(18 \mathrm{p})$. FISH clearly indicated that this $\mathrm{i}(18 \mathrm{p})$ has only the $\mathrm{p}$ arms with a monocentric centromere. This is a case of de novo origin, as none of the other family members showed the presence of $\mathrm{i}(18 \mathrm{p})$. However, as only 50 metaphases were analyzed in each parent, a rare possibility of the presence of mosaics with a very low content of the abnormal cell line in both parents cannot be ruled out, because of the two miscarriages in the mother, which might be due to hidden mosaicism in one of the parents.
One of the causative factors noted for a long time in a series of families with the proband having an additional isochromosome is the maternal meiotic nondisjunction, which occurs due to advanced maternal age (Schinzel 1999). Based on this, there are several contradictory mechanisms to explain the formation of $\mathrm{i}(18 \mathrm{p})$. Eggermann et al. (1997) suggested that formation of $i(18 p)$ is possible only when nondisjunction takes place in meiosis II and centromeric misdivision in a postzygotic mitosis. They exclude the formation of $i(18 p)$ in meiosis I or II, nondisjunction followed by i(18p) formation in meiosis II. Bugge et al. (1996) expressed an opinion that all types of tetrasomy $18 p$ cannot be explained by one mechanism alone, but certain mechanisms are far more likely to occur than others. Schinzel (1999) proposed that the most likely origin of $i(18 p)$ is through maternal meiosis II nondisjunction as a first step, leading to a trisomic zygote, followed by centromeric misdivision or isochromosome formation and loss of the long arm of the additional chromosome. Boyle et al. (2001) explained that $18 p$ can be formed in three ways: (a) an error in meiosis II, in combination with postzygotic misdivision of the centromere; (b) meiosis I nondisjunction followed by post meiotic error; or (c) a post zygotic event.

There are also reports in the Indian context that the somewhat advanced maternal grandmother age (over 30 years) at the time of birth of the mother could be considered as one of the possible risk factors for the occurrence of trisomy 21 in the grandchild apart from the influence of the similar parental age (Malini and Ramachandra 2006). In the present case, the age of the mother (32 years) at the time of conceiving the proband could be considered as advanced and thereby a possible causative factor for $\mathrm{i}(18 \mathrm{p})$ formation. At an advanced age the reproductive system may fail to make the essential proteins like spindle-associated proteins, factors responsible for resting of the oocyte, chiasma-binding proteins, DNA repair enzymes, etc., which are needed for proper meiotic segregation. Therefore, the somewhat advanced maternal age could have lead to nondisjunction and misdivision of chromosome 18 resulting in $\mathrm{i}(18 \mathrm{p})$ in the proband. However, an apparently more probable explanation is the consanguineous marriage between the parents (first-cousin marriage). The mother had already suffered miscarriage twice, during the third month of the second conception and fifth month of the fourth conception, which could be a result 
of the action of recessive genes that had turned out to be lethal. In the proband also there could have been the expression of recessive genes resulting in nondisjunction or misdivision of chromosome 18 , leading to the formation of $\mathrm{i}(18 \mathrm{p})$.

We report here from Mysore, South India, a rare isochromosome $18 \mathrm{p}$ in a female child, which was due to de novo formation. The phenotypic features are mild $(\sim 33 \%)$ when compared to the standard clinical features of tetrasomy $18 \mathrm{p}$. The possible explanation could be the somewhat advanced maternal age at the time of conceiving the proband and/or the consanguineous marriage (first-cousin) between the parents.

Acknowledgments. We thank the family members who have taken part in the present study. Written consent was obtained from the parents for publication of this study. We thank the University of Mysore; the Department of Science and Technology for providing FIST grants to the Department of Zoology; and the Chairman of the Department of Studies in Zoology for giving us (SR) an opportunity to carry out our research activities. We are also grateful to GRC-ICMR (NIRRH) and the Department of Pediatrics, Cheluvamba Hospital, Mysore, for the support and help; to Prof. H.A. Ranganath for his encouragement; as well as to Dr. V.V.N. Gopal Rao, Dr. Mikhil N. Bamne and Ms. Pushpa Saviour who have rendered their immense help for this study.

\section{REFERENCES}

Abeliovich D, Dagan J, Levy A, Steinberg A, Zlotogora J, 1993. Isochromosome 18p in a mother and her child. Am J Med Genet 46: 392-393.

Back E, Toder R, Voiculescu I, Wildberg A, Schempp W, 1994. De novo isochromosome 18p in two patients: Cytogenetic diagnosis and confirmation by chromosome painting. Clin Genet 45: 301-304.

Boyle J, Sangha K, Dill F, Robinson WP, Yong SL, 2001. Grandmaternal origin of an isochromosome $18 \mathrm{p}$ present in two maternal half-sisters. Am J Med Genet 101: 65-69.

Bugge M, Blennow E, Friedrich U, Petersen MB, Pedeutour F, Tsezou A, 1996. Tetrasomy 18p de novo: parental origin and different mechanisms of formation. Eur J Hum Genet 4: 160-167.

Callen DF, Freemantle CJ, Ringenbergs ML, Baker E, Eyre HJ, Romain D, Haan EA, 1990. The isochromosome $18 \mathrm{p}$ syndrome: confirmation of cytogenetic diagnosis in nine cases by in situ hybridization. Am J Hum Genet 47: 493-498.

Eggermann T, Engels H, Moskalonek B, Nothen MM, Muller-Navia J, Schleiermacher E, et al. 1996. Tetrasomy $18 \mathrm{p}$ de novo: identification by FISH with conventional and microdissection probes and analysis of parental origin and formation by short sequence repeat typing. Hum Genet 97: 568-572.

Eggermann T, Engels H, Apacik C, Moskalonek B, Muller-Navia J, Schwanitz G, Stengel-Rutkowski S, 1997. Tetrasomy 18 p caused by paternal meiotic nondisjunction. Eur J Hum Genet 5: 175-177.

Froland A, Holst G and Terslev E, 1963. Multiple anomalies associated with an additional small metacentric chromosome. Cytogenet Cell Genet 2: 99-106.

Fryns Jp, Grubben C, van den Berghe H, 1990. Penile enlargement in tetrasomy $18 \mathrm{p}$ : an additional feature? Ann Genet 33: 239-240.

Fujita K, Fujita HM,1975. An extra small submetacentric chromosome: possible partial 18 trisomy. Jpn J Hum Genet 19: 371-373.

Gocke H, Muradow I, Zerres K, Hansmann M, 1986. Mosaicism of isochromosome 18p: cytogenetic and morphological findings in a male fetus at 21 weeks. Prenat Diagn 6: 151-157.

[ISCN] International System for Human Cytogenetic Nomenclature 1995. Mitelman F, ed. Karger publication.

Irwin DL, Bryan JL, Chan FY, Matthews PL, Healey SC, Peters M, Findlay I, 2003. Prenatal diagnosis of tetrasomy $18 \mathrm{p}$ using multiplex fluorescent PCR and comparison with a variety of techniques. Genetic Testing 7: 1-6.

Kotzot D, Bundschere G, Bernasconi F, Brecevic L, Lurie IW, Basaran S, et al. 1996. Isochromosome $18 \mathrm{p}$ results from maternal meiosis II nondisjunction. Eur J Hum Genet 4: 168-174.

Malini SS, Ramachandra NB, 2006. Influence of advanced age of maternal grandmothers on Down syndrome. BMC Med Genet 7: 4 .

Pinto MR, Fonseca Silva ML, Ribeiro MC, Pina R, 1998. Prenatal diagnosis of mosaicism for tetrasomy 18p: Cytogenetic, FISH and morphological findings. Prenat Diagn 18: 1095-1097.

Rivera H, Moller M, Hernandez A, EnriquezGuerra MA, Arreola R, Cantu JM, 1984. Tetrasomy 18p: a distinctive syndrome. Ann Genet 27: 187-189.

Rothlisberger B, Chrzanowska K, Balmer D, Riegel M, Schinzel A, 2000. A supernumerary marker chromosome originating from two different regions of chromosome 18. J Med Genet 37: 121-124.

Schinzel A, 1999. The impact of nondisjunction in humans: Not only numerical Chromosome aberrations, but more. www.biologia.iniba.it/eca/NEWSLETTER/NS-2/2html

Seabright M, 1971. A rapid banding technique for human chromosomes. Lancet 2: 971-972.

Singer TS, Kohn G, Yatziv S, 1990. Tetrasomy 18p in a child with trisomy 18 phenotype. Am J Med Genet 36: 144-147.

Takeda K, Okamura T, Hasegawa T, 1989. Sibs with tetrasomy $18 \mathrm{p}$ born to a mother with trisomy $18 \mathrm{p}$. J Med Genet 26: 195-197.

Taylor KM, Wolfinger HL, Brown MG, Chadwick Dl, 1975. http://www.cgromosome18org/syndromes.html. 
Origin of a small metacentric chromosome: familial and cytogenetic evidence. Clin Genet 8: 364-369.

Yu LC, Williams J, Wang BB, Vooijs M, Weier HU, Sakamoto M, Ying KL, 1993. Characterization of $\mathrm{i}(18 \mathrm{p})$ in prenatal diagnosis by fluorescence in situ hybridization. Prenat Diagn 13: 355-361. 